\title{
Resistance mechanisms of bacteria to antimicrobial compounds
}

\author{
T.E. Cloete
}

\section{Introduction}

Resistance has been defined as the temporary or permanent ability of an organism and its progeny to remain viable and/or multiply under conditions that would destroy or inhibit other members of the strain. Bacteria may be defined as resistant when they are not susceptible to a concentration of antibacterial agent used in practice. Traditionally, resistance refers to instances where the basis of increased tolerance is a genetic change, and where the biochemical basis is known. Whereas the basis of bacterial resistance to antibiotics is well known, that of resistance to antiseptics, disinfectants and food preservatives is less well understood.

Resistance of biofilm microorganisms has serious economic and environmental implications in many applications like cooling water, papermaking, medical implants, drinking-water distribution, secondary oil recovery,

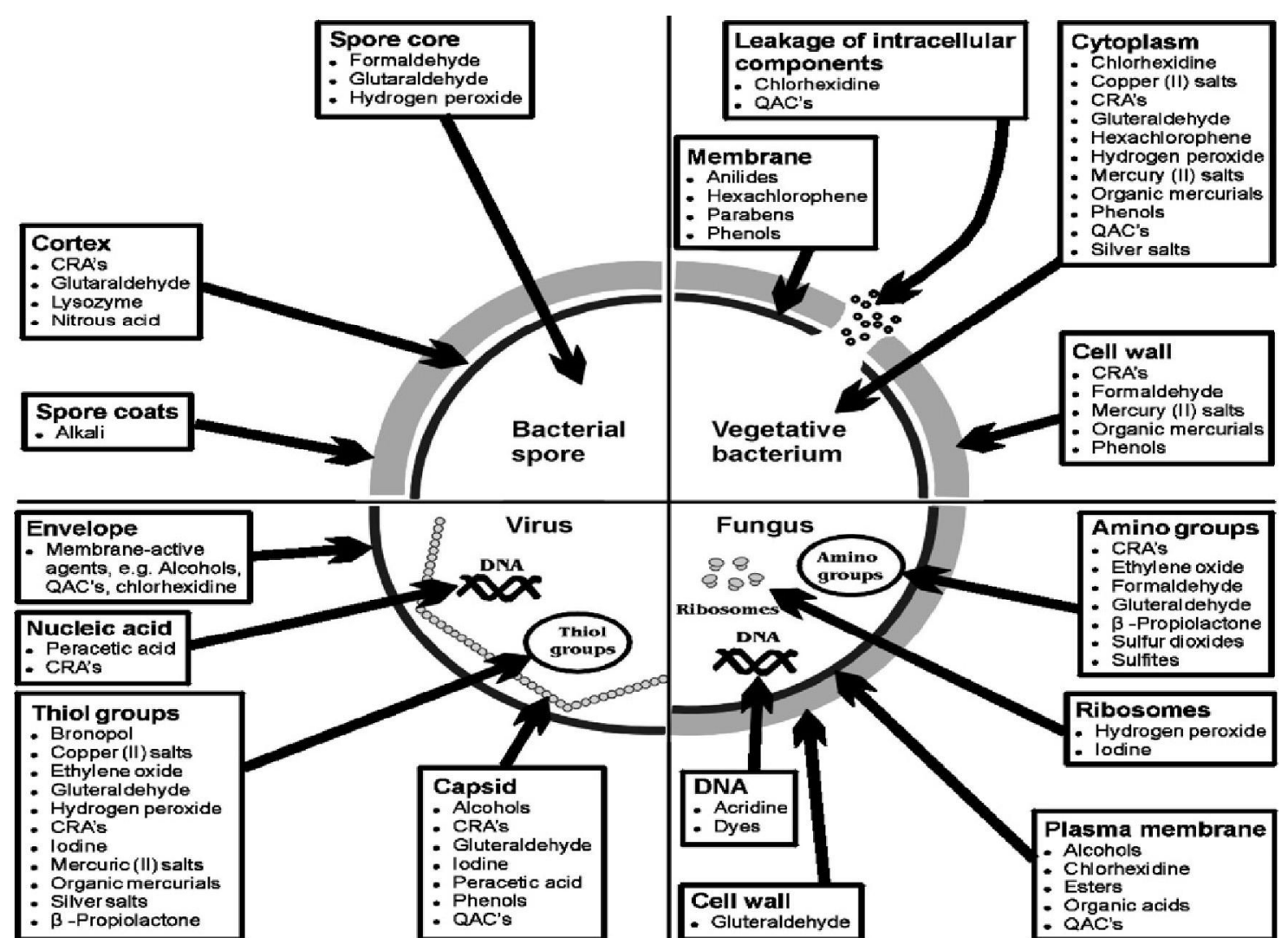

Fig. 1. Mechanism of microorganism inactivation by biocides (after Russel et al., 1997). CRA's = chlorine-releasing agents, QAC's = quaternary ammonium compounds.

metalworking, and food processing (Breyers, 1993; Hoyle and Costerton, 1991). The mechanisms of bacterial attachment and the resulting problems caused in the food and dairy industries are also well known (Carpentier and Cerf, 1993; Criado et al, 1994; Zottola, 1994).

Antimicrobial substances target a range of cellular loci, from the cytoplasmic membrane to respiratory functions, enzymes and the genetic material. However, different bacteria react differently to bactericides, either due to inherent differences such as unique cell envelope composition and non-susceptible proteins, or to the development of resistance, either by adaptation or by genetic exchange. At low concentrations bactericides often act bacteriostatically, and are only bacteriocidal at higher concentrations. 
For bactericides to be effective, they must attain a sufficiently high concentration at the target site in order to exert their antibacterial action.

Possible explanations for the increased resistance of biofilm bacteria include (i) limited diffusion of antimicrobial agents through the biofilm matrix, (ii) interaction of the antimicrobial agents with the biofilm matrix (cells and polymer), (iii) enzyme mediated resistance, (iv) level of metabolic activity within the biofilm, (v) genetic adaptation, (vi) efflux pumps and (vii) outer membrane structure.

This review summarizes the most important mechanisms.

\section{Mechanisms of antimicrobial action}

In order to better understand antimicrobial resistance mechanisms, it is important to know the mechanism of action these compounds (Fig. 1).

\section{Resistance mechanisms}

\subsection{Limited diffusion through the biofilm}

Bacteria growing as adherent biofilms are significantly more resistant towards antimicrobial agents (Korber et al., 1997). Compared with their planktonic counterparts, attached bacteria typically exhibit enhanced resistance to antimicrobial agents (Brown and Gilbert, 1993), biocides (Blenksinsopp et al, 1992), and heat (Frank and Koffi, 1990). Studies have demonstrated the resistance of biofilms of bacteria such as Escherichia coli towards cetrimide (Evans et al, 1990), or Burkholderia cepacia and Serratia marcescens towards biguanides (Marrie and Costerton, 1981; Pallent et al, 1983), isothiazolones and quaternary ammonium compounds (Costerton and Lashen, 1984). Biofilms of other bacterial species also have increased resistance towards biocides such as iodine, iodinepolyvinyl-pyrollidone complexes (Favero et al., 1983), chlorine, monochloramine, peroxygens (Huang et al, 1995a) and Gluteraldehyde (Stewart et al, 1998). Cells of mucoid and non-mucoid Pseudomonas aeroginosa in colonies were at least one-thousandfold less sensitive to the antibiotics tobramycin or cefsulodin than were cells of the same bacteria in suspension. No difference was detected between the mucoid form and the non-mucoid form in the antibiotic sensitivity of colonies, from which it was concluded, that the exopolysaccharide of the mucoid form does not contribute to colony-resistance by forming a barrier to antibiotic diffusion (Nichols et al, 1989).

The proposed mechanism for resistance is that the glycocalyx may create a diffusion barrier to the antimicrobial agent (Brown et al, 1995; de Beer et al, 1994; Chen and Stewart, 1996; Giwercman et al, 1991; Liu et al, 1998; Stewart et al, 1998). Diffusion through a biofilm may be affected by charge (ionic) interactions between the glycocalyx and the antimicrobial agent, by an increase in the distance the agent must diffuse, by molecular sieving (size exclusion), and by the viscosity of the glycocalyx. Some researchers suggest that the polyanionic nature of the glycocalyx creates a barrier (charge interactions) to the diffusion of cationic antimicrobial agents (Chester et al, 1972; Costerton et al, 1981; Costerton and Lashen, 1984). Anderson (1989) suggests that a biofilm, with its thick glycocalyx, must be saturated with an antimicrobial agent before bacteria can be killed. Hence, it was concluded that the glycocalyx matrix contributes to biofilm resistance by cementing cells within the biofilm, anchoring them to one another and to the substratum. The binding of cells within this protective matrix increases the time required to suspend cells in the antimicrobial agent and increases the time required for the antimicrobial agent to contact cells that remain attached in deepest portion of the biofilm. It was concluded that it is not the quantity of glycocalyx that causes resistance in biofilms, but that it is the interaction between the glycocalyx, the cells, the attachment, and the antimicrobial agent that leads to enhanced resistance.

For example, steady-state biofilms were exposed to increasing strengths of sodium hypochlorite (200, 500 and 1000 p.p.m. free chlorine). A two-log decrease in bacterial numbers was achieved at 1000 p.p.m. free chlorine (Matthew et al, 1995). Inplanktonic culture a $100 \%$ kill was achieved when exposed to 10 p.p.m. free chlorine for 30s (Norwood and Gilmour, 2000). Furthermore, the penetration of positively charged hydrophilic drugs such as aminoglycosides and polypeptides was inhibited by the glycocalyx, whilst that of B-lactams, quinolones, and macrolides was not inhibited. Hoyle and Costerton (1991) suggested that the barrier to drug penetration formed by the exopolysaccharide and the low growth rate of bacteria in biofilms were related to drug resistance. In contrast, Nichols et al. (1989) reported that alginate, an exopolysaccharide of mucoid-type $P$. aeroginosa, had a very limited effect in reducing drug penetration. It therefore seems that the mechanism resulting drug resistance on biofilm-forming organisms remains unclear. Nevertheless, limited diffusion is considered one of the most plausible resistance mechanisms associated with biofilms.

\subsection{Enzyme mediated resistance}

Resistance to antimicrobial agents can be due to enzymes transforming the bactericide to a non-toxic form. The 
phenomenon is usually investigated from the biodegradation point of view, i.e. the biodegradation of toxic pollutants. A host of aromatic, phenolic and other compounds, toxic to many bacteria (some of which are employed as bactericides) can be degraded by certain bacteria (Ma et al, 1998).

Examples of enzyme-mediated resistance mechanism include heavy metal resistance and formaldehyde resistance. Resistance to heavy metals includes resistance to the following: mercury, antimony, nickel, cadmium, arsenate, cobalt, zinc, lead, tellurite, copper, chromate and silver. Detoxification is usually by enzymatic reduction of the cation to the metal, whereas some heavy metal resistance genes are carried on plasmids, whilst others are chromosomal. The resistant phenotype is usually inducible by the presence of the heavy metal. Some heavy metals induce resistance to a broader spectrum of heavy metals. Arsenate, arsenite and antimony, for example, induce resistance to each other in $E$. coll

Studies on the detoxification of formaldehyde by $P$. aeruginosa and $P$. putida indicated that formaldehyde is reduced by an NAD+-gluthathione-dependent dehy-drogenase, giving formaldehyde NAD+oxidoreductase. This enzyme is probably plasmidencoded, and appears to be constitutively expressed. Resistance to most formaldehyde-releasing formaldehyde condensates is also due to formaldehyde dehydrogenase activity as the antibacterial mechanism of these condensates appears to be via formaldehyde (Kummerle et al, 1996).

\subsection{Interaction and neutralization of the antimicrobial substance by the biofilm}

The initial stage of bactericide action is binding to the bacterial cell surface after which it must traverse the cell wall (gram positive) or outer membrane (gram negative) to reach its site of action at the cytoplasmic membrane or cytoplasm. In gram positive bacteria there are no specific receptor molecules or permeases to assist or block bactericide penetration. Intrinsic resistance of gram positive bacteria to bactericides is therefore low. The gram negative cell envelope has, however, evolved to regulate the passage of substances into and out of the cell to a remarkable degree of specificity. All the components of the cell envelope except peptidoglycan play a role in the barrier mechanisms because peptidoglycan is spongy and therefore permeable. $P$. aeruginosa is the most resistant non-sporeforming bacteria to most bactericides, due to the superior barrier properties of its outer membrane. In a recent study, the antimicrobial activity of a series of new 2-arylthio- $\mathrm{N}$-alkylmaleimides were compared and many were found active against Staphylococcus aureus, Bacillus subtilis and $E$. coli. Only one of the 51 derivatives tested was marginally active against $P$. aeruginosa.

Another possible mechanism for biofilm resistance is that the glycocalyx matrix in a biofilm reacts with and neutralizes the antimicrobial agent (Brown et al, 1995). For example, it has been suggested that iodine reacts with glycocalyx compounds, since the interaction of iodine with other organic molecules such as proteins, fatty acids, sulfhydryl compounds and vitamin $\mathrm{C}$ has been documented (Alexander, 1983; McAvoy et al, 1989). These interactions involve oxidation of the organic molecule by iodine (Alexander, 1983; McAvoy et al, 1989). Characklis and Dydek (1976) have furthermore demonstrated that chemical interactions between chlorine and biofilm glycocalyx occur and that the glycocalyx material creates a chlorine demand.

The glycocalyx is a polyanionic polymer and acts as an exchange resin. It quantitatively adsorbs biocide protecting the bacterial cell from biocide action. Gram negative bacteria growing in biofilms have a higher ratio of unsaturated to saturated fatty acids and a higher ratio of $\mathrm{C}_{16}$ to $\mathrm{C}_{18}$ fatty acids. Resistant bacteria show similar changes in membrane-lipid profiles. Surface hydrophobicity of quaternary ammonium compounds (QAC) and amphoteric resistant cells was higher than that of unadapted cells. Biofilm bacteria often have a higher surface hydrophobicity due to attachment structures.

Research has supported the theory that organic material is somehow attracted to the glycocalyx. At least some of these molecules must diffuse to and into the microorganisms embedded in the glycocalyx to facilitate the observed growth. Nonoxidising biocides, being organic molecules of small to intermediate size would also associate favourably with the glycocalyx. At least some would diffuse to and into the microorganisms embedded in the glycocalyx and exert their antibacterial activity. The mechanism of increased resistance must be related to altered surface properties of cells growing in the biofilm environment.

\subsection{Metabolic state of the organisms in the biofilm}

Resistance of biofilms towards antimicrobial agents has been explained by the imposition of slow, biofilm-specific growth within the biofilm (Gilbert et al, 1989; Huang et al., 1995b). The physiological state of cells and the nature of the habitat can lead to considerable variation in the susceptibility of bacteria to bactericides. The composition of the bacterial cell envelope does change as a response to available or limiting nutrients, so that the barrier properties of the envelope are affected. Exposure to sub-inhibitory concentrations of bactericides can lead to phenotypic adaptation, resulting in a resistant cell population. In $E$. coli certain proteins induced by heat or starvation stress also confer resistance to $\mathrm{H}_{2} \mathrm{O}_{2}$ and to UV light. Most bactericideresistance is due to adaptation, and the resistant phenotype is mostly lost upon removal of the bactericide.

It has also been suggested that biofilm reduced susceptibility requires that at least some of the cells within a biofilm experience a nutrient limitation that causes them to enter a slow-growing or starved state (Brown et al, 1988). Antimicrobial treatment of biofilms resulted in cells near the biofilm-bulk fluid interface losing their respiratory activity first, whilst respiratory 
activity persisted deep in the biofilm-bulk fluid interface. Slow or non-growing cells are less susceptible to a variety of antimicrobial agents when compared with cells grown in rich media at high specific growth rates. There is considerable experimental support for this mechanism (Gilbert and Brown, 1995) and also for striking spatial heterogeneity in the physiological status of bacteria within relatively thick (100 um) biofilms (Wentland et al, 1996; Xu et al, 1998).

\subsection{Genetic adaptation}

Reduced biofilm susceptibility, by genetic adaptation would require that at least some of the cells in a biofilm adopt a distinct, and relatively protected, biofilm phenotype. The practical importance of this postulated mechanism, is significant because it implies that reduced susceptibility of biofilm bacteria is genetically programmed.

The multiple antibiotic resistance (mar) operon is a global regulator controlling the expression of various genes in $E$. coli which constitutes the mar leads to a multi-drug resistant phenotype, which includes resistance towards structurally unrelated antibiotics, organic solvents and the disinfectant pine oil (Maira-Litran et al, 2000).

The development of resistance to oxidising bactericides has not been reported in the biofouling control literature. However, a variety of bacteria, mostly fermentative, exhibit oxidising stress response by producing oxidant-degrading and repair enzymes. Stress response means that cells become more resistant to a deleterious factor within hours of exposure to subinhibitory quantities of the factor. A variety of defense genes have been characterised in $E$. coli, encoding various superoxide dismutases, catalysts, alkyl hydroperoxide reductases and glutathione reductases, as well as DNA repair enzymes (Farr and Kogoma, 1991). In addition various regulatory genes have been characterised, including oxy $R$ and sox $R$. These regulators determine intra-cellular redox potential, and activate stress response when cells are exposed to oxidising agents.

\subsection{Outer membrane structure}

Resistance to antimicrobial agents can be due to a mechanism of adaptation of the cell envelope. For bactericides to be effective, they must be able to penetrate the cell envelope and attain a sufficiently high concentration at the target site to exert their antibacterial action. Hydrophilic antibacterial agents are primarily prevented from entering through the outer membrane by the lipopolysaccharide layer and the underlying phospholipids, whereas hydrophobic agents are excluded by outer membrane proteins. Certain antibiotic-resistant bacterial strain either lack or overexpress certain outer membrane proteins. An example is a strain of $P$. aeroginosa resistant to the antibiotic imipenem. This strain lacks Opr $D$, a porin selective for certain carbon sources.

Studies on the resistance mechanism of Pseudomonas aeroginosa to isothiazolone indicated that a $35 \mathrm{kDa}$ outer membrane protein was detectable in wild cells and not in resistant cells (Brozel and Cloete, 1994). It was proposed that this protein was the port of entry for isothiazolone.

Resistance and the development thereof in $P$. aeroginosa to the bactericide sodium dimethyldithiocarbamate (SMT) was investigated. Following $24 \mathrm{~h}$ of exposure to SMT, $P$. aeroginosa had an altered profile of outer membrane proteins as determined by SDS-PAGE. Resistant cells had a further altered profile. Resistance of $P$. aeruginosa is ascribed to a change in the outer membrane protein profile, leading to improved exclusion of SMT (Brozel and Cloete, 1993).

\subsection{Efflux pumps}

Multidrug efflux pumps can pump out a wide range of seemingly dissimilar compounds (Nikaido, 1996; Paulsen et al, 1996). An example is the QAC efflux system of Staphylococcus aureus which is coded for by two gene systems. The genes qacA and qacB encode for a high level of resistance, and qacC and qacD encode for a low level of resistance. qacC and qacD are further identical to the ebr gene encoding for resistance to ethidium bromide in $S$. aureus, explaining why resistance to $Q A C$ is often concurrent with resistance to ethidium bromide. The qacA gene codes for a $50 \mathrm{kDa}$ protein which mediates energydependent efflux of both benzalkonium chloride and ethidium bromide. The qac $\mathrm{C}$ gene also mediates energy-dependent efflux of benzalkonium chloride and ethidium bromide. Two different but isofunctional gene systems appear to have evolved in $S$. aureus (Littlejohn et al, 1990; Rouche et al, 1990).

Three such efflux systems have also been described for $P$. aeroginosa, MexA-MexB-OprM, MexC-MexD-OprJ and MexEMexF-OprN, respectively (Schweizer, 1998). The MexAB-OprM system appears to mediate efflux of a variety of compounds (tetracycline, chloramphenicol, fluoroquinonoles, B-lactams (except carbapenems), novobiocin, erythromycin, fusidic acid, rifampin, etc. 


\section{References}

Alexander, N.M., 1983. Reaction of povidone-iodine with amino acids and other important biological compounds. In: Digenis, G.A., Ansell, J. (Eds.), In: Proceedings of the International Symposium on Povidone. University of Kentucky Press, Lexington, pp. 274-288.

Anderson, R.L., 1989. lodophor antiseptics: intrinsic microbial contamination with resistant bacteria. Infection Control and Hospital Epidemology 10 , 443-446.

Blenksinsopp, S.A., Khoury, A.E., Costerton, J.W., 1992. Electrical enhancement of biocide efficacy against Pseudomonas aeroginosa biofilms. Applied and Environmental Microbiology 58, 3770-3773.

Breyers, J.D., 1993. Bacterial biofilms. Current Opinion in Biotechnology 4, 197-204.

Brown, M.R.W., Gilbert, W., 1993. Sensitivity of biofilms to antimicrobial agents. Journal of Applied Bacteriology 74, 87-97.

Brown, M.R.W., Allison, D.G., Gilbert, P., 1988. Resistance of bacterial biofilms to antibiotics: a growth rate related effect?. Journal of Antimicrobial Chemotherapy 22, 777-783.

Brown, M.L., Henry, Aldrich, H.C., Gauther, J.J., 1995. Relationship between glycocalyx and povidence-iodine resistance in Pseudomonas aeroginosa (ATCC 27853) biofilms. Applied and Environmental Microbiology 61, 187-193.

Brözel, V.S., Cloete, T.E., 1993. Resistance of Pseudomonas aeroginosa to sodium dimethyldithiocarbamate by adaptation. Current Microbiology $26,275-280$.

Brözel, V.S., Cloete, T.E., 1994. Resistance of Pseudomonas aeroginosa to isothiazolone. Journal of Applied Bacteriology 76, $576-582$.

Carpentier, B., Cerf, O., 1993. Biofilms and their consequences, with particular reference to hygiene in the food industry. Journal of Applied Bacteriology 75 , 499511.

Characklis, W.G., Dydek, S.T., 1976. The influence of carbon-nitrogen ratio on the chlorination of microbial aggregates. Water Research $10,512-522$.

Chen, X., Stewart, P.S., 1996. Chlorine penetration into artificial biofilm is limited by a reaction-diffusion interaction. Environmental Science Technology $30,2078-$ 2083.

Chester, I.R, Gray, G.W., Wilkinson, S.G., 1972. Further studies of the chemical composition of the lipopolysaccharide of Pseudomonas aeroginosa. Biochemical Journal 126, 395-407.

Costerton, J.W., Lashen, E.S., 1984. Influence of biofilm on efficacy of biocides on corrosion-causing bacteria. Materials Performance 23, $13-17$.

Costerton, J.W., Irwin, R.T., Cheng, K.J., 1981. The bacterial glycocalyx in nature and disease. Annual Reviews in Microbiology 35, $299-324$.

Criado, M.T., Suarez, B., Ferreiros, 1994. The importance of bacterial adhesion in the dairy industry. Food Technology 48, $123-126$.

de Beer, D., Srinivasan, R, Steward, P.S., 1994. Direct measurement of chlorine penetration into biofilms during disinfection. Applied Environmental Microbiology $60,4339-4344$.

Evans, D.J., Allison, D.G., Brown, M.R.W., Gilbert, P., 1990. Growth rate and the resistance of Gram-negative biofilms towards cetrimide USP. Journal of Antimicrobial Chemotherapy 26, 473-478.

Farr, S.B., Kogoma, T., 1991. Oxidative stress response in Escherichia coli and Salmonella typhimurium. Microbiological Review 55, $1309-1316$.

Favero, M.S., Bond, W.W., Peterson, N.J., Cook, E.H., 1983. Scanning electron microscopic study of bacteria resistant to iodophor solutions. In: Proceedings of the International Symposium on Povidone. University of Kentucky, Lexington, USA, pp. 158-166.

Frank, J.F., Koffi, R.A., 1990. Surface-adherent growth of Listeria monocytogenes is associated with increased resistance to sanitizers and heat. Journal of Food Protection 53, 550-554.

Gilbert, P., Brown, M.R.W., 1995. Mechanisms of the protection of bacterial biofilms from antimicrobial agents. In: Lappin-Scott, H., Costerton, J.W. (Eds.), Microbial Biofilms. Cambridge University Press, Cambridge, pp. 118-130.

Gilbert, P., Allison, D.G., Evans, D.J., Handley, P.S., Brown, M.R, 1989. Growth rate control of adherent bacterial populations. Applied Environmental Microbiology $55,1308-1311$.

Giwercman, B., Jensen, E.T., Hoiby, N., Kharazmi, A., Costerton, J.W., 1991. Induction of B-lactamase production in Pseudomonas aeroginosa biofilms. Antimicrobial Agents and Chemotherapy 35, 1008-1010.

Huang, C.T., Yu, F.P., McFeters, G.A., Stewart, P.S., 1995a. Non-uniform spatial patterns of respiratory activity within biofilms during disinfection. Applied and Environmental Microbiology 61, 2252-2256.

Huang, C.T., Philip, Yu, F.P., McFeters, G.A., Stewart, P.S., 1995b. Nonuniform spatial patterns of respiratory activity within biofilms during disinfection. Applied and Environmental Microbiology 61, 2252-2256.

Hoyle, B.D., Costerton, J.W., 1991. Bacterial resistance to antibiotics: the role of biofilms. Progress in Drug Research 37, 91-105.

Korber, D.R., Choi, A., Wolfaardt, G.M., Ingham, S.C., Caldwell, D.E., 1997. Substratum topography influences susceptibility of Salmonella enteritidis biofilms to trisodium phosphate. Applied and Environmental Microbiology 63, 3352-3358.

Kummerle, N., Feucht, H.H., Kaulfers, P.M., 1996. Plasmid-mediated formaldehyde resistance in Escherichia col: characterization of resistance gene. Antimicrobial Agents and Chemotherapy 40, 2276-2279.

Littlejohn, T.G., DiBeradino, D., Messerotti, L.J., Spiers, S.J., Skurray, R.A., 1990. Structure and evolution of a family of genes encoding antiseptic and disinfectant resistance in Staphylococcus aureus. Gene 101, 59-66.

Liu, X., Roe, F., Jesaitis, A., Lewandowski, Z., 1998. Resistance of biofilms to the catalyst inhibitor 3-amino 1,2,4-triazole. Biotechnology and Bioengineering 59, 156-162.

Ma, J.F., Hager, P.W., Howell, M.L., Phibbs, P.V., Hassett, D., 1998. Cloning and characterization of the Pseudomonas aeroginosa zwf gene encoding glucose-6phosphate dehydrogenase, an enzyme important in resistance to methyl viologen (paraquat). Journal of Bacteriology 180, $1741-1749$.

Maira-Litran, T., Allison, D.G., Gilbert, P., 2000. Expression of the multiple antibiotic resistance operon (mar) during growth of Escherichia coli as a biofilm. Journal of Applied Microbiology 88, 243-247.

Marrie, T.J., Costerton, J.W., 1981. Prolonged survival of Serratia marcescens in chlorhexidine. Applied and Environmental Microbiology 42, $1093-1102$.

McAvoy, M.J., Newton, V., Paull, J., Morgan, P., Gacesa, P., Russell, N.J., 1989. Isolation of mucoid strains of Pseudomonas aeroginosa from noncystic-fibrosis patients and characterization of the structure of their secreted alginate. 28, 183-189.

Nichols, W.W., Evans, M.J., Slack, M.P.E., Walmsley, H.L., 1989. The penetration of antibiotics into aggregates of mucoid and non-mucoid Pseudomonas aeroginosa. Journal of General Microbiology 135, 1291-1303.

Nikaido, H., 1996. Multidrug efflux pumps of gram-negative bacteria. Journal of Bacteriology 178, 5853-5859.

Norwood, D.E., Gilmour, A., 2000. The growth and resistance to sodium hypochlorite of Listeria monocytogenes in a steady-state multispecies biofilm. Journal of Applied Microbiology 88, 512-520. 
Pallent, L.J., Hugo, W.B., Grant, D.J.W., Davies, A., 1983. Pseudomonas cepacia as contaminant and infective agent. Journal of Hospital Infection 4, 13-15.

Paulsen, I., Brown, M.H., Skurray, R.A., 1996. Proton-dependent multidrug efflux systems. Microbiological Review 60, 575-608.

Rouche, D.A., Cram, D.S., Di Benardino, D., Littlejohn, T.G., Skurray, R.A., 1990. Efflux-mediated antiseptic gene qacA from Staphylococcus aureus: common ancestry with tetracycline and sugar-transport proteins. Molecular Microbiology 4, 2051-2062.

Russel, A.D., Furr, R.J., Maillard, J.Y., 1997. Microbial susceptibility and resistance to biocides. ASM News 63, $481-487$.

Schweizer, H.P., 1998. Intrinsic resistance to inhibitors of fatty acid biosynthesis in Pseudomonas aeroginosa is due to efflux: application of a novel technique for generation of unmarked chromosomal mutations for the study of efflux systems. Antimicrobial Agent and Chemotherapy 42, $394-398$.

Stewart, P.S., Grab, L., Diemer, J.A., 1998. Analysis of biocide transport in an artificial system. Journal of Applied Microbiology 85, 495-500.

Wentland, E.J., Stewart, P.S., Huang, C.T., McFeters, G.A., 1996. Spatial variations in growth rate within Klebsiella pneumoniae colonies and biofilm. Biotechnological Progress 12, 316-321.

Xu, K.D., Stewart, P., Xia, F., Huang, C.-T., McFeters, G.A., 1998. Spatial physiological heterogeneity in Pseudomonas aeruginsoa biofilm is determined by oxygen available. Applied and Environmental Microbiology 64, 4035-4039.

Zottola, E.A., 1994. Microbial attachment and biofilm formation: a new problem in the food industry? Food Technology $48,107-114$. 